А. А. Маляренко (Міжнар. мат, центр НАН Українн, Київ)

\title{
ЛОКАЛЬНІ ВЛАСТИВОСТІ ГАУССОВИХ ВИПАДКОВИХ ПОЛІВ НА КОМПАКТНИХ СИМЕТРИЧНИХ ПРОСТОРАХ I ТЕОРЕМИ ТИПУ ДЖЕКСОНА ТА БЕРНШТЕИНА
}

We consider local properties of sample functions of Gaussian isotropic random fields on the compact Riemann symmetric spaces $\mathcal{M}$ of rank one. We give conditions under which the sample functions of a field almost surely possess logarithmic and power modulus of continuity. As a corollary, we prove the Bernshtein-type theorem for optimal approximations of functions of this sort by harmonic polynomials in the metric of space $L_{2}(\mathcal{M})$. We use the Jackson-Bernshtein-type theorems to obtain sufficient conditions of almost surely belonging of the sample functions of a field to classes of functions associated with Riesz and Cesàro means.

Розглядаються локалыі властивості вибіркових функціи гауссових ізотропинх випапкових полів на компахтиих ріманових симетричних просторах $\mathcal{M}$ рангу 1 . Наведено умови, при виконанні яких вибіркові фуцкції поля манже напевие мають логарифмічния та степеневий модулі неперервності. Як наслідок доведено теорему типу Бернштейна для оптимальних наближень таких функцін гармонічними многочленами в метриці простору $L_{2}(M)$. Теореми типу Джексона - Бернштейıа використано для огримания достатніх умов належності маиже напевıе нибіркових функцій до класів фуцкцій, пов'язаиих з середніми Рісса та Чезаро.

1. Вступ. Нехай $\mathcal{M}=G / K-N$-вимірний компактний симетричний простір рангу 1 з групою рухів $G$ і стаціонарною підгрупою $K[1]$. Нехай $\xi(x)-$ неперервне в середньому квадратичному гауссове поле на $\mathcal{M}$ з нульовим середнім значенням і кореляційною функцією

$$
B(x, y)=E \xi(x) \overline{\xi(y)} .
$$

Поле $\xi(x)$ називається ізотропнияь в широкольу розумінні [2], якщо

$$
B(g x, g y)=B(x, y), \quad g \in G, \quad x, y \in \mathcal{M} \text {. }
$$

В теоремі 1 наводимо достатні умови неперервності випадкового поля $\xi(x)$, що узагальнюють результати роботи [3], в якій відповідні твердження доведено для випадку $N$-вимірної сфери $\mathscr{M}=S^{N}$.

Використовуючи поняття моделі випадкової функції та ядра гауссової міри в просторі $C(\mathcal{M})$ неперервних функцін на $\mathcal{M}[4]$, доводимо теорему типу Бернштейна (теорема 2): якшо середня квадратична похибка оптимального наближення функції гармонічними многочленами порядку не більше $n$ мажорується числовою послідовністю $a_{n}$, що задовольняе одну з умов (9а) (9c), то функція задовольняе відповідну умову (11a) - (11c).

В пункті 3 використовуємо теореми типу Джексона та Бернштейна з [5] для отримання достатніх умов належності майже напевне вибіркових функцій випадкового поля $\xi(x)$ до класів неперервних функцій, пов'язаних з середніми значеннями Рісса та Чезаро (теорема 3 ).

2. Умови неперервності випадкового поля на компактному симетричному просторі і теорема типу Бернштейна. Нехай $\rho(x, y)$ - довжина геодезичної лінії, що зв' язує точки $x, y \in \mathcal{M}, L=\sup _{x, y \in \mathcal{M}} \rho(x, y)$ - діаметр простору $\mathcal{M}$. 3 метою спрошення подальших формул зафіксуємо одиницю вимірювання відстані в метриці $\rho$ так, щоб виконувалась умова $L=\pi$.

Існує список компактних ріманових симетричних просторів рангу 1 [1]. А came: 
сфери

$$
S^{N}=S O(N+1) / S O(N), \quad N \geq 1 ;
$$

дійсні проективні простори

$$
P^{N}(\mathbb{R})=S O(N+1) / O(N), \quad N \geq 2 ;
$$

комплексні проективні простори

$$
P^{N}(\mathbb{C})=S U(l+1) / S(U(l) \times U(1)), \quad l=\frac{N}{2} \geq 2
$$

кватерніонні проективні простори

$$
P^{N}(\mathbb{H})=S p(l+1) / S p(l) \times S p(1), \quad l=\frac{N}{4} \geq 2 ;
$$

проективна площина Келі над алгеброю октав

$$
P^{16}(\text { Cay })=F_{4(-52)} / S O(9),
$$

де $S O(m)$ - група ортогональних матрищь порядку $m$ з одиничним визначником; $O(m)$ - група ортогональних матриць порядку $m ; S U(m)-$ група унітарних матриць порядку $m$ з одиничним визначником; $U(m)$ - група унітарних матриць порядку $m ; S(U(l) \times U(1))$ - підгрупа матрищь з одиничним визначником в групі $U(l) \times U(1) ; S p(m)$ - група ортогональних симплектичних матриць порядку $m ; F_{4(-52)}-$ виняткова група Лі рангу 4 розмірності 52.

Нехаи $S_{\theta}(x)$ - множина точок простору $\mathcal{M}$, що знаходяться на віддалі $\theta$ від фіксованої точки $x \in \mathcal{M}, A(\theta)-(N-1)$-вимірна міра Лебега многовиду $S_{\theta}(x)$. Має місце формула [6]

$$
A(\theta)=\Omega_{N} 2^{p} \sin ^{p}\left(\frac{\theta}{2}\right) \sin ^{q} \theta,
$$

де $\Omega_{N}-N$-вимірна міра Лебега сфери одиничного радіуса в просторі $\mathbb{R}^{N}$, а параметри $p$ та $q$ мають наступні значення: для сфер $p=0, q=N-1$; для дійсних проективних просторів $p=N-1, q=0$; для комплексних проективних просторів $p=N-2, q=1$; для кватерніонних проективних просторів $p=N-4$, $q=3$ і для проективної площини Келі $p=8, q=7$. Диференціальний оператор

$$
\Delta_{0}=\frac{1}{A(\theta)} \frac{d}{d \theta}\left(A(\theta) \frac{d}{d \theta}\right), \quad 0<\theta<\pi,
$$

являя собою радіальну частину оператора Лапласа - Бельтрамі простору $\mathcal{M}$.

Нехай $d m-G$-інваріантна міра на $\mathcal{M}$, нормована умовою $\int_{\mathcal{M}} d m=1$. Тоді спектр оператора $\Delta_{0}$ в просторі $L^{2}(\mathcal{M}, d m)$ складається з точок $-\lambda_{n}^{2}=$ $=-n(n+\alpha+\beta+1), n \geq 0$, де параметри $\alpha$ та $\beta$ обчислюються за формулами

$$
\alpha=\frac{1}{2}(p+q-1), \quad \beta=\frac{1}{2}(q-1) \text {. }
$$

Розглянемо неперервне унітарне зображення $U$ групи $G$ в просторі $L^{2}(\mathcal{M}$ $d m)$, шо діє за формулою 


$$
(U f)(g)(x)=f\left(g^{-1} x\right), \quad g \in G .
$$

Легко перевірити, що оператор $\Delta_{0}$ комутує із зображенням $U$. Тому згідно з загальною теорією [7], в кожному 3 просторів

$$
\mathcal{H}_{n}=\left\{f \in C^{\infty}(\mathcal{M}): \Delta_{0} f=-\lambda_{n}^{2} f\right\}, \quad n \geq 0,
$$

реалізується незвідна компонента $U_{n}$ зображення $U$, причому всі компоненти попарно нееквівалентні. Елементи простору $\mathcal{H}_{n}$ називаються гармонічними льногочленахии.

Теорема Петера - Вейля [8] стверджує, що кожне з названих зображень $є$ зображенням класу 1 відносно підгрупи $K$, тобто містить ненульові $K$ інваріантні вектори. Розмірність простору таких векторів дорівнюе кратності входження $U_{n}$ до $U$, в нашому випадку - одиниці. Зафіксуємо $K$-інваріантний вектор $\Psi$ одиничної довжини. Матричний елемент зображення $U_{n}$, що вімповідає вектору $\Psi$ (зональна сферична функція)

$$
\Psi_{n}(g)=\int_{\mathcal{M}} \Psi\left(g^{-1} y\right) \overline{\psi(y)} d m(y),
$$

не залежить від вибору вектора $\psi$. Більш того, вона залежить від відстані між точками $x$ та 0 з простору $\mathcal{M}$, що відповідають елементу $g \in G$ та одиничному елементу $e \in G$ при канонічній проекції з $G$ на $\mathscr{M}$. В [9] доведено, що зональна сферична функція компактного ріманового простору рангу 1 має вигляд

$$
\psi_{n}(\theta)=\frac{P_{n}^{(\alpha, \beta)}(\cos \theta)}{P_{n}^{(\alpha, \beta)}(1)}
$$

де $P_{n}^{(\alpha, \beta)}(\cos \theta)$ - многочлен Якобі.

Позначимо через $h(\mathcal{M}, n)$ розмірність простору $\mathscr{H}_{n}$. Нехай $\left\{\psi_{l}: 1 \leq l \leq\right.$ $\leq h(\mathcal{M}, n)\}, \psi_{1}=\psi-$ фіксований ортонормований базис у просторі $\mathcal{H}_{n}$. Згідно 3 теоремою Петера - Вейля функціі

$$
S_{n}^{l}(x)=\sqrt{h(\mathcal{M}, n)} \int_{G} \Psi_{l}\left(g^{-1} x\right) \overline{\Psi_{l}(x)} d g
$$

утворюють ортонормований базис простору $L^{2}(\mathcal{M})$. Тут $d g$ - имовірнісна міра Хаара на $G$. Маемо

$$
\sum_{l=1}^{h(\mathcal{M}, n)} S_{n}^{l}(x) \overline{S_{n}^{l}(y)}=h(\mathcal{M}, n) \Psi_{n}(\rho(x, y))
$$

(це просто формула множення матриць). Нехай тепер $b_{n} n \geq 0$ - послідовність невід'ємних дійсних чисел, що задовольняють умову

$$
\sum_{n=0}^{\infty} h(\mathcal{M}, n) b_{n}<\infty
$$

а $\left\{\xi_{n}^{l}: n \geq 0,1 \leq l \leq h(\mathcal{M}, n)\right\}-$ стандартна гауссова послідовність. Тоді гауссове випадкове поле 


$$
\xi(x)=\sum_{n=0}^{\infty} \sqrt{b_{n}} \sum_{l=1}^{h\left(\mathscr{M}_{,}, n\right)} S_{n}^{l}(x) \xi_{n}^{l}
$$

буде ізотропним, а һ̆ого корелящін̆на функція, обчнслена з застосуванням теореми Карунена і формули (2), матиме вигляд

$$
B(x, y)=\sum_{n=0}^{\infty} h(\mathcal{M}, n) b_{n} \psi_{n}(\rho(x, y)) .
$$

3 результатів роботи [10] випливає, що формула (3) описує всі можливі кореляційні функції ізотропних випадкових полів на $\mathcal{M}$

Доведемо допоміжне твердження, що узагальнює тауберову теорему М. Й. Ядренка [3] (розділ II, теорема 9) для випадку компактних симетричних просторів рангу 1.

Лема 1. Нехай $\gamma(t):[0,+\infty) \mapsto[0,+\infty)$-фуниія, цо задовольняе умови:

$$
\begin{aligned}
& \lim _{t \rightarrow+\infty} \gamma(t)=+\infty \\
& \text { функція } \frac{t^{2}}{\gamma(t)} \text { не спадає, починаючи з деякого } t \\
& \sum_{n=0}^{\infty} h(\mathcal{M}, n) b_{n} \gamma(n)<+\infty
\end{aligned}
$$

Тоді при $\theta \downarrow 0$ виконується співвідношення

$$
B(1)-B(\cos \theta)=O\left(\gamma^{-1}\left(\theta^{-1}\right)\right) .
$$

Доведення. Метод доведення часткового випадку леми 1 для сфер, запропонований в [3], дослівно переноситься на загальнин випадок, за винятком одного твердження. А саме: у випадку сфери виконуетъся нерівність

$$
1-\Psi_{n}(\theta) \leq \frac{1}{2} n^{2} \theta^{2}
$$

3 метою доведення аналогічної нерівності в загальному випадку із заміною коефіцієнта $\frac{1}{2}$ на коефіцієнт, що залежить лише від $\alpha$ та $\beta$, перепишемо (4) у вигляді

$$
1-\frac{P_{n}^{(\beta, \beta)}(\cos \theta)}{P_{n}^{(\beta, \beta)}(1)} \leq \frac{1}{2} n^{2} \theta^{2}, \quad \beta=\frac{N-2}{2} .
$$

Безпосередній підрахунок за формулами (1) показує, що для всіх компактних ріманових симетричних просторів рангу 1, крім сфер, виконується нерівність $\alpha>\beta>-1$. У роботі [2] доведено, що для таких значень параметрів $\alpha$ та $\beta$ вірна формула

$$
\frac{P_{n}^{(\alpha, \beta)}(t)}{P_{n}^{(\alpha, \beta)}(1)}=\sum_{k=0}^{n} a_{k n} \frac{P_{k}^{(\beta, \beta)}(t)}{P_{k}^{(\beta, \beta)}(1)}
$$

при цьому коефіцієнти $a_{k n}$ невід'ємні. При $t=1$ одержимо $\sum_{k=0}^{n} a_{k n}=1$. Тому маємо 


$$
1-\frac{P_{n}^{(\alpha, \beta)}(\cos \theta)}{P_{n}^{(\alpha, \beta)}(1)}=\sum_{k=0}^{n} a_{k n}\left(1-\frac{P_{k}^{(\beta, \beta)}(\cos \theta)}{P_{k}^{(\beta, \beta)}(1)}\right) \leq \frac{1}{2} \theta^{2} \sum_{k=0}^{n} k^{2} a_{k n}
$$

(використано нерівність (5)). Для обчислень суми в правій частині останнъоі формули візьмемо похідну від обох частин (6). Використовуючи формулу (4.21.7) з роботи [11] і рівність

$$
P_{n}^{(\alpha, \beta)}(1)=\frac{\Gamma(\alpha+n+1)}{n ! \Gamma(\alpha+1)}
$$

одержуємо

$$
\frac{n(n+\alpha+\beta+1)}{\alpha+1}=\frac{1}{\beta+1} \sum_{k=0}^{n}\left[k^{2}+(2 \beta+1) k\right] a_{k n},
$$

звідки

$$
\sum_{k=0}^{n} k^{2} a_{k n} \leq \frac{\beta+1}{\alpha+1} n(n+\alpha+\beta+1) \leq \frac{(\alpha+\beta+2)(\beta+1)}{\alpha+1} n^{2}
$$

і остаточно

$$
1-\frac{P_{n}^{(\alpha, \beta)}(\cos \theta)}{P_{n}^{(\alpha, \beta)}(1)} \leq \frac{(\alpha+\beta+2)(\beta+1)}{2 \alpha+2} n^{2} \theta^{2},
$$

що й доводить лему.

Теорема 1. Нехай виконується одна з умов

$$
\begin{gathered}
\sum_{n=0}^{\infty} h(\mathcal{M}, n) b_{n} \ln ^{2 \varepsilon}(n+1)<+\infty, \quad \varepsilon>0, \\
\sum_{n=0}^{\infty} h(\mathcal{M}, n) b_{n} n^{2 \delta} \ln (n+1)<+\infty, \quad 0<\delta \leq 1, \\
\sum_{n=0}^{\infty} h(\mathcal{M}, n) b_{n} n^{2 \delta}<+\infty, \quad 0<\delta \leq 1 .
\end{gathered}
$$

Тоді існуе додатна мьайже напевне скінченна випадкова величина $C(\omega)$, для якої відповідно

$$
\begin{gathered}
|\xi(x)-\xi(y)| \leq \frac{C(\omega)}{|\ln \rho(x, y)|^{\varepsilon}}, \\
|\xi(x)-\xi(y)| \leq \frac{C(\omega)}{(\rho(x, y))^{\gamma}}, \quad \gamma<\delta, \\
|\xi(x)-\xi(y)| \leq \frac{C(\omega)}{(\rho(x, y))^{\gamma} \sqrt{|\ln \rho(x, y)|}}, \quad \gamma<\delta .
\end{gathered}
$$

Доведения. Імплікащії (7a) $\Rightarrow(8 \mathrm{a})$, (7b) $\Rightarrow(8 \mathrm{~b})$ та $(7 \mathrm{c}) \Rightarrow(8 \mathrm{c})$ доводяться так само, як і в [3] (теореми 10,11 та 12 розділу 2), із заміною посилання на теорему 9 цікі ж роботи посиланням на лему 1.

Нехай $\mathcal{P}_{n}$ - пряма сума просторів $\mathcal{H}_{k}, 0 \leq k \leq n$. Позначимо через $E_{n}^{(2)}(f)$ похибку оптималного наближення функції $f \in L^{2}(\mathcal{M}, d m)$ елементами простору $\mathcal{P}_{n}$ в нормі простору $L^{2}(\mathcal{M}, d m)$ : 


$$
E_{n}^{(2)}(f)=\inf _{p_{n} \in P_{n}}\left\|f-p_{n}\right\|_{2} \text {. }
$$

Теорема 2. Нехай $f \in L^{2}(\mathcal{M}, d m), a_{n}, n \geq 0-$ послідовність додатних чисел, що монотонно прялуує до нуля $і$ задовольняе одну з улюв

$$
\begin{gathered}
a_{n-1}^{2}-a_{n}^{2}=O\left(\frac{1}{n^{2+\zeta} h(\mathcal{M}, n) \ln ^{2 \varepsilon}(n+1)}\right), \quad \varepsilon>0, \\
a_{n-1}^{2}-a_{n}^{2}=O\left(\frac{1}{n^{2+\zeta+2 \delta} h(\mathcal{M}, n) \ln (n+1)}\right), \quad 0<\delta \leq 1, \\
a_{n-1}^{2}-a_{n}^{2}=O\left(\frac{1}{n^{2+\zeta+2 \delta} h(\mathcal{M}, n)}\right), \quad 0<\delta \leq 1 .
\end{gathered}
$$

Якщио

$$
E_{n}^{(2)}(f)=O\left(a_{n}\right)
$$

то функція $f$ задовольняе відповідну умову

$$
\begin{gathered}
|f(x)-f(y)| \leq \frac{C}{|\ln \rho(x, y)|^{\varepsilon}}, \\
|f(x)-f(y)| \leq \frac{C}{(\rho(x, y))^{\gamma}}, \quad \gamma<\delta, \\
|f(x)-f(y)| \leq \frac{C}{(\rho(x, y))^{\gamma} \sqrt{|\ln \rho(x, y)|}}, \quad \gamma<\delta .
\end{gathered}
$$

Зауваження 1. В теорії наближення функцій умови виду (10) називаються конструктивнильи характеристикальи функції, а умови виду (11) - дескриптивнияи характеристикалєи.

Доведення теореми 2. Доведемо лише імплікацію (9a) $\Rightarrow$ (11a), інші імплікації доводяться аналогічно.

Нехай $\xi(x)$ - гауссове випадкове поле на $\mathcal{M}$, для якого

$$
b_{n}=\frac{1}{n^{1+\zeta / 2} h(\mathcal{M}, n) \ln ^{2 \varepsilon}(n+1)} .
$$

Нехай $\mathbf{P}$ - гауссова міра в просторі $C(\mathcal{M})$, що відповідає випадковому полю $\xi(x)$. Згідно 3 теоремою 1 множина функцін, що задовольняють умову (11a), має P-міру 1. Це означає, що кожен елемент лінійного носія міри $\mathbf{P}$, тобто найменшого замкнутого підпростору Р-міри 1 , також задовольняє умову (11a).

3 іншого боку, згідно з [4] (розділ 9, теорема 6), лінійний носій міри $\mathbf{P}$ співпадає з замиканням ї ядра $H_{\mathbf{P}}$, тобто множини функцій $f \in C(\mathscr{M})$, для яких мipa

$$
\mathbf{P}_{f}(A)=\mathbf{P}(\{g \in C(\mathcal{M}): f+g \in A\})
$$

абсолютно неперервна відносно міри Р. Залишається довести, що кожна функція $f$, що задовольняє умову (10), належить до $H_{\mathbf{P}}$.

Нехай $\mathcal{A}=\{(n, l): n \geq 0,1 \leq l \leq h(\mathcal{M}, n)\}, \mathfrak{A}$ - $\sigma$-алгебра всіх підмножин множини $\mathcal{A}, \mathrm{v}$ - міра на $\mathfrak{A}$, що визначається умовою $\mathrm{v}(n, l)=b_{n}$. Тоді 
функції $m_{x}(n, l)=S_{n}^{l}(x)$ утворюють льдель випадкового поля $\xi(x)$ [4], тобто виконуеться умова

$$
E \xi(x) \overline{\xi(y)}=\int_{\Re} m_{x}(n, l) \overline{m_{y}(n, l)} d v(n, l)
$$

(це просто інша форма запису співвідношення (3)). Згідно з [4] (розділ 9, формула 17), образ оператора $I$ з $L^{2}(\mathcal{A}, v)$ в $C(\mathcal{M})$, шо діє за формулою

$$
I\left(\left\{c_{n}^{l}\right\}\right)=\sum_{n=0}^{\infty} b_{n} \sum_{l=1}^{h\left(\mathcal{M}_{1} n\right)} c_{n}^{l} S_{m}^{l}(x)
$$

співпадає з $H_{\mathbf{P}}$. Це означає, що простір $H_{\mathbf{P}}$ складається з функцій, у яких коефіцієнти Фур'є $d_{n}^{l}$ за ортонормованим базисом $\left\{S_{n}^{l}\right\}$ задовольняють умову

$$
\sum_{n=0}^{\infty} b_{n}^{-1} \sum_{l=1}^{h(\mathcal{M}, n)}\left|d_{n}^{l}\right|^{2}<+\infty
$$

Похибка $E_{n}^{(2)}(f)$ обчислюеться за формулою

$$
E_{n}^{(2)}(f)=\sqrt{\sum_{k=n+1}^{\infty} \sum_{l=1}^{h(\mathcal{M}, n)}\left|d_{n}^{l}\right|^{2}},
$$

звідки одержуємо

$$
\sum_{l=1}^{h(\mathcal{M}, n)}\left|d_{n}^{l}\right|^{2}=O\left(a_{n-1}^{2}-a_{n}^{2}\right)=O\left(n^{-1-\zeta / 2} b_{n}\right),
$$

а це і означає збіжність ряду (12).

3. Умови належності вибіркових функцій до класів Рісса та Чезаро. Для формулювання результату цього пункту необхідно навести деякі означення з [5]. Нехай $Y_{n}$-оператор ортогонального проектування з $L^{2}=L^{2}(\mathcal{M}, d m)$ на $\mathcal{H}_{n}, r, R, \delta$ - фіксовані додатні числа. Оператор $S_{R}^{r} \delta$, дія якого задається формулою

$$
S_{R}^{r, \delta} f(x)=\sum_{n: \lambda_{n}<R}\left(1-\left(\frac{\lambda_{n}}{R}\right)^{r}\right)^{\delta} Y_{n} f(x),
$$

називається середнім Рісса функції $f$. Якщо іскує функція $g \in L^{2}$, яка задовольняє умову

$$
Y_{n} g=\lambda_{n}^{r} Y_{n} f, \quad n \geq 0,
$$

то вона називається похідною Рісса порлдку $r$ і позначається $g=\Lambda^{r} f$. Простір

$$
W^{2, r}=\left\{f \in L^{2}: \Lambda^{r} f \in L^{2}\right\}
$$

називається простором Рісса порядку $r$. Функціонал

$$
K\left(f, t ; l^{2}, W^{2, r}\right)=\inf _{g \in W^{2, r}}\left(\|f-g\|_{2}+t\left\|\Lambda^{r} g\right\|_{2}\right)
$$

називається $K$-функціоналом ПIтррі. 
Нехай $d \mu_{s}-(N-1)$-вимірна міра Лебега на сфері $S_{\theta}(x)$. Число

$$
\tau_{\theta}(f ; x)=\frac{1}{A(\theta)} \int_{S_{\theta}(x)} f(y) d \mu_{s}(y)
$$

називається сферичних середнім функпії $f$, а число

$$
\omega_{r}\left(f, \delta ; L^{2}\right)=\sup _{0<\theta \leq \delta}\left\|\left(\tau_{\theta}-I\right)^{r / 2} f\right\|_{2}
$$

- $\overline{\mathbf{1 1}}$ льдулем гладкості. Oператор $C_{n}^{\delta}$, дія якого задається формулою

$$
C_{n}^{\delta} f=\frac{1}{A_{n}^{\delta}} \sum_{k=0}^{n} A_{n-k}^{\delta} Y_{k} f, \quad A_{n}^{\delta}=\frac{\Gamma(n+\delta+1)}{n ! \Gamma(\delta+1)},
$$

називається середнім Чезаро функції $f$.

Нехай тепер $\mathcal{K}_{\gamma}$ - замикання в $C(\mathcal{M})$ множини функцій, що задовольняють одну з умов

$$
\begin{array}{cc}
\left\|S_{R}^{r, \delta} f-f\right\|_{2}=O\left(R^{-\gamma}\right), & R \uparrow \infty, \\
K\left(f, t ; L^{2}, W^{2, r}\right)=O\left(t^{\gamma}\right), & t \downarrow 0, \\
\omega_{r}\left(f, \delta ; L^{2}\right)=O\left(\delta^{\gamma}\right), & \delta \downarrow 0, \\
\left\|C_{n}^{\delta} f-f\right\|_{2}=O\left(n^{-\gamma}\right), \quad n \uparrow \infty,
\end{array}
$$

причому в перших трьох умовах $r-$ довільне натуральне число і $0<\gamma<r$, а в останнің умові $0<\gamma<1$.

Теорема 3. Нехай $\xi(x)-$ гауссове ізотропне випадкове поле на $\mathcal{M}$ і виконано умови (7а) та

$$
b_{n}=O\left(\frac{1}{(n-1)^{2 \gamma}}-\frac{1}{n^{2 \gamma}}\right)
$$

Тоді вибіркові функції випадкового поля $\xi(x)$ майже напевне належать до кожного з класів $\mathcal{K}_{\gamma}$.

Доведення. За теоремою 1 вибіркові функції поля $\xi(x)$ неперервні, тому будь-який елемент ядра відповідної гауссової міри має вигляд

$$
f(x)=\sum_{n=0}^{\infty} b_{n} \sum_{l=1}^{h(\mathcal{M}, n)} c_{n}^{l} S_{n}^{l}(x), \quad \sum_{n=0}^{\infty} b_{n} \sum_{l=1}^{h\left(\mathcal{M}_{,}, n\right)}\left|c_{n}^{l}\right|^{2}<\infty .
$$

Згідно з [5] (теореми 3.1, 3.2, 5.1, 5.2), кожна з умов (13) рівносильна умові

$$
E_{n}^{(2)}(f)=O\left(n^{-\gamma}\right) \text {. }
$$

Залишається довести, що кожна з функщіи (14) задовольняє умову (15).

Справді, умова (15) рівносильна тахій умові на коефіцієнти Фур'є функції $f$.

$$
\sum_{l=1}^{h(\mathcal{M}, n)}\left|d_{n}^{l}\right|^{2}=O\left(\frac{1}{(n-1)^{2 \gamma}}-\frac{1}{n^{2 \gamma}}\right)
$$


а для коефіцієнтів Фур'є функції (14) маємо

$$
\sum_{l=1}^{h(\mathcal{M}, n)}\left|d_{n}^{l}\right|^{2}=b_{n} \sum_{l=1}^{h(\mathcal{M}, n)} b_{n}\left|c_{n}^{l}\right|^{2}=O\left(b_{n}\right)
$$

оскільки послідовність $\sum_{l=1}^{h(\mathcal{M}, n)} b_{n}\left|c_{n}^{l}\right|^{2}$ членів збіжного ряду збігаєтъся до нуля і тому обмежена.

1. Хелзасои C. Дифференциальная геометрия и симметрические пространства. - М.: Наука, 1964. $-533 \mathrm{c}$.

2. Askey $R$. Orthogonal polynomials and special functions. - Philadelphia: Soc. Industr. and Appl. Math., 1975. -110 p.

3. Ядренко М. К. Спектральная теория случайых полей. - Киев: Выща шк., 1980, - 203 с.

4. Лифшиц М. А. Гауссовские случайые функции. - Кнев: ТВиМС, 1995. -210 с.

5. Li L Riesz. Means on compact Riemanian symmetric spaces // Math. Nachr. - 1994. - 168. P. 227-242.

6. Askey R., Bingham R. H. Gaussian processes on compact symmetric spaces // Z. Wahrscheinlichkeitstheor. und verw. Geb. - 1976. - 37. - P. 127-143.

7. Виленкин Н.Я. Специалыне фуикцин и теория представлеший групп. - М.: Наука, 1991. $576 \mathrm{c}$.

8. Желобенко Д. П. Компактиые группы Ли и их представления. - М.: Наука, 1970. - 664 с.

9. Gangolli $R$. Positive definite functions on certain homogeneous spaces, and certain stochastic processes related to Levy's Brownian motion of several parameters // Ann. Inst. H. Poincaré. B. 1967. - 3. - P. 121-226.

10. Yaglom A. M. Second-order homogeneous random fields. // Proc. 4th Berkeley Symp. Math. Statist. and Probab. - 1960. - 2. - P. 593-622.

11. Сеzе $Г$. Ортогоналыные многочлешы. - М.: Физматтиз, 1962. - 500 с.

Одержан 17.09 .96 